\title{
Measurement of Vibrational Non-equilibrium in a Supersonic Freestream using Dual-Pump CARS
}

\author{
Andrew D. Cutler ${ }^{*}$, Gaetano Magnotti ${ }^{\dagger}$, Luca M. L. Cantu ${ }^{\dagger}$, Emanuela C. A. Gallo ${ }^{\dagger}$ \\ The George Washington University, Newport News, VA 23602 \\ Paul M. Danehy ${ }^{\ddagger}$ and Rob Baurle ${ }^{\S}$ \\ NASA Langley Research Center, Hampton, VA 23681 \\ Robert Rockwell $^{* *}$, Christopher Goyne ${ }^{\dagger \dagger}$, and Jim McDaniel ${ }^{*}$ \\ University of Virginia, Charlottesville, VA 22904
}

\begin{abstract}
Measurements have been conducted at the University of Virginia Supersonic Combustion Facility of the flow in a constant area duct downstream of a Mach 2 nozzle, where the airflow has first been heated to approximately $1200 \mathrm{~K}$. Dual-pump CARS was used to acquire rotational and vibrational temperatures of $\mathrm{N}_{2}$ and $\mathrm{O}_{2}$ at two planes in the duct at different downstream distances from the nozzle exit. Wall static pressures in the nozzle are also reported. With a flow of clean air, the vibrational temperature of $\mathbf{N}_{2}$ freezes at close to the heater stagnation temperature, while the $\mathrm{O}_{2}$ vibrational temperature is about $1000 \mathrm{~K}$. The results are well predicted by computational fluid mechanics models employing separate "Iumped" vibrational and translational/rotational temperatures. Experimental results are also reported for a few percent steam addition to the air and the effect of the steam is to bring the flow to thermal equilibrium.
\end{abstract}

\section{Introduction}

$\mathrm{H}$ YPERSONIC flow simulation facilities provide high-speed, high-enthalpy flow conditions to test aerospace vehicles, thrusters and engines. The test gas is typically heated to avoid freestream condensation, to allow combustion tests in engines and/or to simulate high enthalpy "real-gas" effects such as dissociation. The test gas is also accelerated through converging-diverging nozzles to provide the required flow velocity. However, heating and expanding the test gas imparts non-ideal characteristics to the gas. For example, combustion-heated facilities can have 10's of percent of water in the freestream while shock- or arc-heated facilities have a few percent of nitric oxide (NO) present in the test gas. This contamination is known as free stream vitiation. Other imperfect attributes of gases produced in hypersonic facilities include the existence of atomic oxygen and nitrogen from the dissociation of $\mathrm{O}_{2}$ and $\mathrm{N}_{2}$ respectively, or copper or iron eroding from facility electrodes or facility walls. Yet another imperfect characteristic that the gas can acquire in hypersonic test facilities is vibrational non-equilibrium. This phenomenon often occurs when a high-temperature stagnant gas is expanded suddenly through a converging-diverging nozzle to supersonic or hypersonic velocity. At first, the expansion occurs slowly enough (high enough collision frequency) to keep the rotational and translational modes of diatomic molecules in equilibrium with each other. However, as the gas passes down the nozzle, the expansion can become too great (too low collision frequency) and the flow speed too fast for vibrational modes to stay in equilibrium with the rotational and translational modes of the gas. ${ }^{1}$ The number of collisions required for the rotational mode to be brought into equilibrium with the translational mode is

\footnotetext{
* Professor, Mechanical and Aerospace Engineering Department, 1 Old Oyster Point Road, Suite 200. Associate

Fellow AIAA

${ }^{\dagger}$ Graduate Student. AIAA Student Member

* Research Scientist, Advanced Sensing and Optical Measurement Branch, MS 493. Associate Fellow AIAA

$\S$ Aerospace Engineer, Hypersonic Air Breathing Propulsion Branch, MS 169. Associate Fellow AIAA

** Senior Scientist. Member AIAA

${ }^{\dagger}$ Research Associate Professor. Associate Fellow AIAA

$\$$ Professor. Associate Fellow AIAA
} 
much less than required to bring the vibrational mode into equilibrium, so the rotational mode is normally assumed to be in equilibrium. Consequently the Boltzmann distribution of population among rotational states, resulting in a rotational temperature, $T_{r}$, is out of equilibrium with the distribution among vibrational states, $T_{v}$. Further complicating this situation, different molecules $\left(\mathrm{N}_{2}, \mathrm{O}_{2}\right.$, NO, etc.) can develop different vibrational temperatures. These non-equilibrium effects may be important because they can affect ignition or reaction rates in supersonic combustion engines tested in ground-based facilities. Ignition could be affected because molecules in excited vibrational states may be more reactive than those in ground vibrational states. Vibrational non-equilibrium also changes the static pressure measured at the exit of a nozzle because it changes the effective specific heat capacity of the expansion process. In order to compare computational fluid dynamics (CFD) calculations with experiments, it is critically important to model or to determine the magnitude of these non-ideal characteristics. Non-equilibrium effects can be computed but measurements should be made, if possible, to validate the computations.

Vibrational non-equilibrium in hypersonic facilities has been measured previously in arc jets using spontaneous Raman spectroscopy, ${ }^{2}$ and in shock tunnels using nitric oxide planar laser induced fluorescence (NO PLIF) ${ }^{3}$ and broadband $\mathrm{N}_{2}$ coherent anti-Stokes Raman spectroscopy (CARS). ${ }^{4}$ It has been included in the calculation of facility freestreams and shock-layer flows. ${ }^{5}$ This phenomenon has been studied analytically or through correlations by numerous researchers. ${ }^{1,6}$ Vibrational non-equilibrium has been predicted to occur in electrically heated blowdown wind tunnels. ${ }^{78}$ In this paper, the dual-pump coherent anti-Stokes Raman spectroscopy (DP-CARS) technique is used to study the vibration-rotation non-equilibrium in the supersonic nozzle expansion at the University of Virginia Supersonic Combustion Facility (UVaSCF). DP-CARS is a non-intrusive, laser-based technique, originally developed by Lucht, ${ }^{9}$ that has been implemented by several researchers to measure temperature and species concentration in scramjet combustors. ${ }^{10,11,12}$ At the NASA Langley Research Center, the DP-CARS technique was successfully used to measure temperature, $\mathrm{N}_{2}, \mathrm{O}_{2}$ and $\mathrm{H}_{2}$ concentration in scramjet engines. ${ }^{13}$

In a previous paper, we reported DP-CARS measurements in the UVa dual-mode scramjet "Configuration A."14 We observed vibrational non-equilibrium in the spectra of air in the freestream of this experiment, particularly for the $\mathrm{N}_{2}$. We noted that, according to Landau-Teller theory, ${ }^{1}$ the relaxation time for pure $\mathrm{N}_{2}$ is roughly $28 \mathrm{~ms}$ at the nozzle exit condition while the flow travels the length of the scramjet in only about $1 \mathrm{~ms}$. Thus, the potential observation of vibrationally frozen flow was deemed plausible.

In the present paper, we report a more detailed analysis of vibrational non-equilibrium in the UVa facility. The experimental data in this paper were acquired in two separate test entries. The first is the aforementioned entry in which measurements were made in Configuration A. The second occurred in the spring of 2012 using Configuration C. The facility operating conditions were the same in both configurations but the model geometry was different. Configuration A consisted of two sections of duct downstream of the nozzle: a Combustor section followed by an Extender section. For this configuration, the CARS data presented herein were obtained just downstream of the nozzle exit. Configuration $\mathrm{C}$ consisted of Isolator, Combustor, TDLAT (enables tunable diode laser absorption tomography measurements), and Extender sections downstream of the nozzle. For this configuration, the measurement plane was just downstream of the exit of the constant area Isolator section. Consequently, measurements at the same operating conditions allow the vibrational relaxation in the isolator duct to be studied. Also, for the Configuration $\mathrm{C}$ cases, various levels of steam were added. Addition of steam was expected to bring the gas flow back towards equilibrium. ${ }^{15}$ Computations of the flowfield for both equilibrium and thermal nonequilibrium have been performed and are compared with the data. Our contribution is to demonstrate that vibrational non-equilibrium can be found in supersonic nozzles as well as hypersonic nozzles, and show that published modeling methods can predict it.

\section{Experimental Facility}

The UVaSCF is a direct-connect electrical-resistance-heated facility capable of providing continuous, clean air flow with a total temperature of $1200 \mathrm{~K}$. In the current experiments, the facility was operated at a total pressure of $296 \mathrm{kPa}$ with an air flow rate of $0.18 \mathrm{~kg} / \mathrm{s}$ issuing through a Mach 2 facility nozzle. These conditions simulate a scramjet vehicle traveling at Mach 5 with engine inlet compression slowing the flow to Mach 2 at the entrance to the engine isolator. Details of the general tunnel operation are described in Refs. 16 and 17. The experiments were performed with no fuel flow and no combustion in the scramjet flowpath.

The facility has the capability of simulating the operation of a combustion-heated facility through the addition of steam, carbon dioxide, and supplemental oxygen that is injected into the air flow upstream of the electric heater. ${ }^{17}$ The flow rates of each gas are metered via choked orifices. Stagnation pressure and temperature are measured upstream of each orifice and, together with a measured orifice diameter and assumed discharge coefficient, this 
provides sufficient information to determine the mass flow rate of the added gases. Injection upstream of the facility heater promotes full mixing and heating of the added gases. Only steam has been added for the experiments described here.

The water vapor is supplied by an electric boiler that can generate steam continuously at pressures up to approximately $3500 \mathrm{kPa}$. The generated steam travels through plumbing to a three-way valve upstream of the tunnel that allows steady-state operation of the steam system and heater to be achieved before the steam is introduced into the main air flow. This is important as the air temperature at the point of steam injection must be sufficiently high to prevent water condensation. A combination of heat from the facility heater core and external steam lines can raise the incoming air temperature to $360 \mathrm{~K}$ to allow up to $15 \% \mathrm{H}_{2} \mathrm{O}$ by mole fraction without condensation.

Measurements were performed at three different levels of water vapor addition: $1.9 \%, 3.7 \%$, and $5.7 \%$. Sources of uncertainty in the steam delivery system include the discharge coefficient and throat area of the choked orifice as well as instrumentation uncertainty associated with measuring pressure and temperature. In addition, the steam generator itself may not maintain a perfectly constant steam pressure and temperature with time. The steam injection system has been previously verified and the uncertainty quantified as $3.4 \%$ of the steam flow rate. ${ }^{18}$

The Mach 2 facility nozzle is a two-dimensional, symmetrical, contoured nozzle with an area ratio of 1.786 . The contour in the vicinity of the throat consists of two circular arcs that join tangentially at the area minimum. The radii of the arcs downstream and upstream of the minimum, normalized by the half height of the nozzle throat $(7.11 \mathrm{~mm})$, are 5.38 and 9.38, respectively. Downstream of the throat, the nozzle is contoured to straighten the flow. The side walls of the nozzle are parallel and the exit is $4 h$ in height and $6 h$ between the side walls, where $h=6.35 \mathrm{~mm}$ is the reference length in this experiment (equal to the height of the fuel injector).

The dual-mode scramjet consists of a length of constant area duct matched to the nozzle exit, followed by a diverging duct with a fuel injector. CARS measurements may be made at a series of cross stream planes using specially designed windows in the two sidewalls. Each incorporates a $3.18 \mathrm{~mm}$ wide slot that spans the entire height of the duct, with an airtight enclosure and offset (to avoid laser beam damage) windows at Brewster's angle. This enables CARS measurements over the entire cross plane at each axial station. The measurements reported herein all pertain to the most upstream plane, which was located at the beginning of the combustor section just prior to the start of the divergence in the duct. For the purposes of this paper, the only significant difference between Configurations $\mathrm{A}$ and $\mathrm{C}$ is that $\mathrm{C}$ has an extra constant area length of duct (the "isolator") located between the combustor and the nozzle, whereas A does not. Consequently, for A the CARS measurement plane is located at $x / h$ $=13.8$ from the nozzle throat, whereas for $\mathrm{C}$ it is at $x / h=55.6$.

\section{Dual-Pump CARS Technique}

Coherent anti-Stokes Raman spectroscopy (CARS) is a non-linear spectroscopic technique in which three laser beams-two pump beams (green and yellow) and a Stokes beam (red)-are focused and crossed at their focal point, and a fourth signal laser beam (blue) is generated at the intersection through a four-wave mixing process, as illustrated in Fig. 1 $(\mathrm{a}, \mathrm{b})$. Resonances associated with Raman-active molecular transitions between rotationalvibrational states strengthen the CARS signal in a manner dependent on gas composition and temperature. The wavelength of excitation-or "Raman shift" -corresponds to the difference in energy between the pump beam photon and the Stokes beam (longer wavelength) photon. In the broadband CARS technique, the Stokes laser beam is spectrally broad whereas the pump beams are spectrally narrow, and this enables multiple resonances to be excited simultaneously. The signal beam is spectrally broad and carries with it the spectral signature of the gases present (over some range of wavelengths). In the dual-pump technique, two CARS processes (in which the roles of the pump beams, $\omega_{p 1}$ and $\omega_{p 2}$, are exchanged) take place simultaneously, and the signals from these two processes are superimposed. This signal beam is dispersed by a spectrometer and spectra are compared to analytical spectral models to obtain information on composition and temperature. 

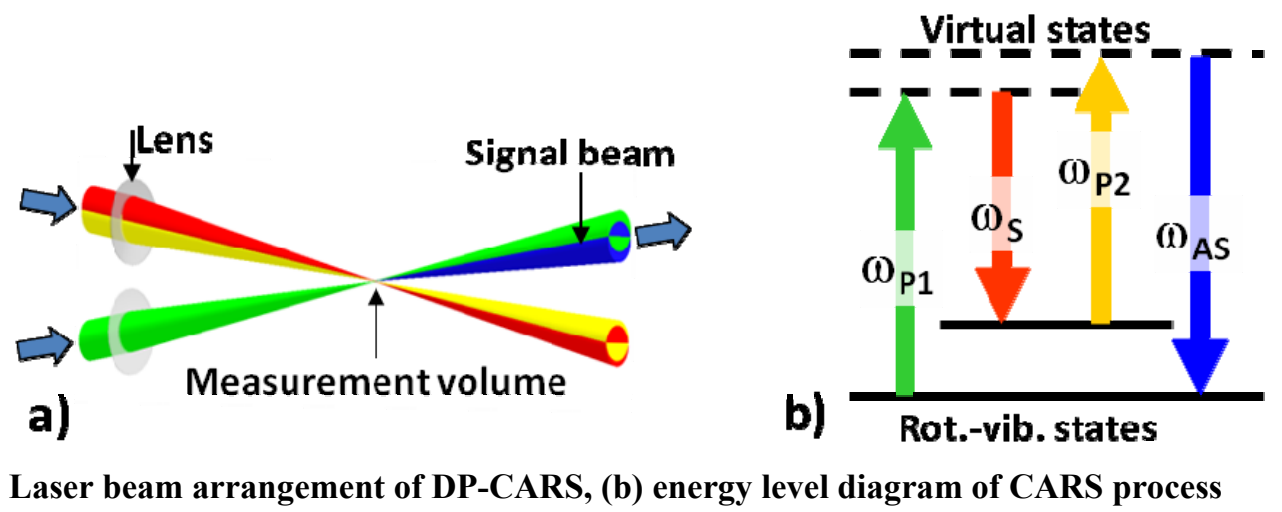

Figure 1. (a) Laser beam arrangement of DP-CARS, (b) energy level diagram of CARS process

The CARS system is the same as described in Refs. 14 and 19. An injection seeded Nd:YAG laser, frequency doubled to $532 \mathrm{~nm}$; a narrow band dye laser (Spectra Physics PDL-2) operating at $551 \mathrm{~nm}$; and a home-built broadband dye laser centered at $603 \mathrm{~nm}$ with FWHM of $10 \mathrm{~nm}$, are used to generate the CARS signal. The pulse width of the lasers is about $8 \mathrm{~ns}$ and the repetition rate is $20 \mathrm{~Hz}$. The green output of the Nd:YAG laser, approximately $900 \mathrm{~mJ}$ per pulse, is divided three ways: $170 \mathrm{~mJ}$ to pump the broadband dye laser, $415 \mathrm{~mJ}$ for the narrowband dye laser, and $280 \mathrm{~mJ}$ for the CARS green beam. The two dye lasers are combined using a dichroic mirror. The three lasers are located on a mobile cart, and the beams are relayed to the measurement volume through several relay mirrors. The beams are focused by a $75 \mathrm{~cm}$ focal length spherical lens for the superimposed dye laser beams and a $60 \mathrm{~cm}$ lens for the green beam. At the measurement volume, the three beams interact and generate the signal beam containing a broad CARS spectrum centered at $490 \mathrm{~nm}$. The laser energies at the measurement volume are 63,32 and $19 \mathrm{~mJ}$ for the Nd:YAG, the narrowband and the broadband beams respectively. The lens focusing the green beam is tilted with respect to the incoming beam so that, by introducing astigmatism, it shapes the focal spot at the beam crossing to a roughly $4: 1$ axis ratio ellipse. This helps in reducing beam steering effects in flows with large, unsteady density gradients. ${ }^{20}$ A series of dichroic mirrors and filters is used to separate the signal from the $\mathrm{Nd}$ :YAG beam. The signal is then focused by a spherical lens on the input slit of a one meter spectrometer with a 2400 grooves $/ \mathrm{mm}$ grating. The CARS signal is dispersed by the spectrometer and recorded on a cooled, backilluminated CCD camera. In the implementation at UVa, the laser beams are generated on a mobile cart that is located in a lab adjacent to the scramjet. Beams are relayed to the measurement plane in the facility via a series of dichroic mirrors and a translation system that enables the measurement point to be moved in the flow, and the signal is relayed by additional mirrors from the measurement volume to the detection system located on an optical bench in the scramjet lab. ${ }^{14}$

The collected spectra are analyzed by comparison with the Sandia CARSFT code ${ }^{21}$ and using the fitting algorithm developed by Cutler. ${ }^{22}$ The CARSFT code contains all the theoretical models for calculating the spectra, including the models for computing the CARS theoretical susceptibility and convolutions with laser line shape and instrument function. CARSFT is capable of either comparing with or fitting to an experimental spectrum, but the algorithm converges poorly (or not at all) with more than a couple of fitted parameters at a time. When handling large data sets, especially when fitting for both temperature and several species mole fractions, the Cutler code is used. This code interpolates from previously-generated libraries of spectra computed using CARSFT, and fits for experimental parameters that may vary from spectrum to spectrum (amplitude scaling and horizontal shift) as well as the temperature and species mole fractions. The library contains spectra at various temperatures and species compositions and is sparsely structured so that its size scales to a power less than the number of species plus one. ${ }^{22}$ Generally, it is assumed that not all the species present in the gas being probed appear in the CARS spectrum, and that the nonresonant susceptibility of the species not present (the buffer gas) is constant and known. In this work, both $\mathrm{N}_{2}$ and $\mathrm{O}_{2}$ Q-branches appear in the spectrum so both major species in air are present. We use the Voigt line shape $\operatorname{model}^{23}$ and take the pressure (which affects the spectra through the line shape) to be the pressure measured at the edge of the flow by wall static pressure taps. In the current paper, air is treated as a single species variable in the library (consisting of 0.79 mole fraction $\mathrm{N}_{2}$ and 0.21 mole fraction $\mathrm{O}_{2}$ ) while $\mathrm{H}_{2} \mathrm{O}$ is the buffer gas and the library has two variables (temperature and mole fraction of air). If only air is present, the mole fraction fits to 1.0 give or take an error associated with errors in the spectral modeling or assumed pressure.

The CARSFT code was modified for this work to permit the calculation of spectra with different rotational and vibrational temperatures of $\mathrm{N}_{2}$ and $\mathrm{O}_{2}$ and comparison to experiment, but was not modified to fit for vibrational 
temperatures. The Cutler et al. ${ }^{22}$ fitting code was not able to fit for separate vibrational temperatures either, although it could employ libraries in which the vibrational temperature was set to a specified constant or set equal to the rotational. Specific fitting strategies are discussed in the Results section.

\section{Computations}

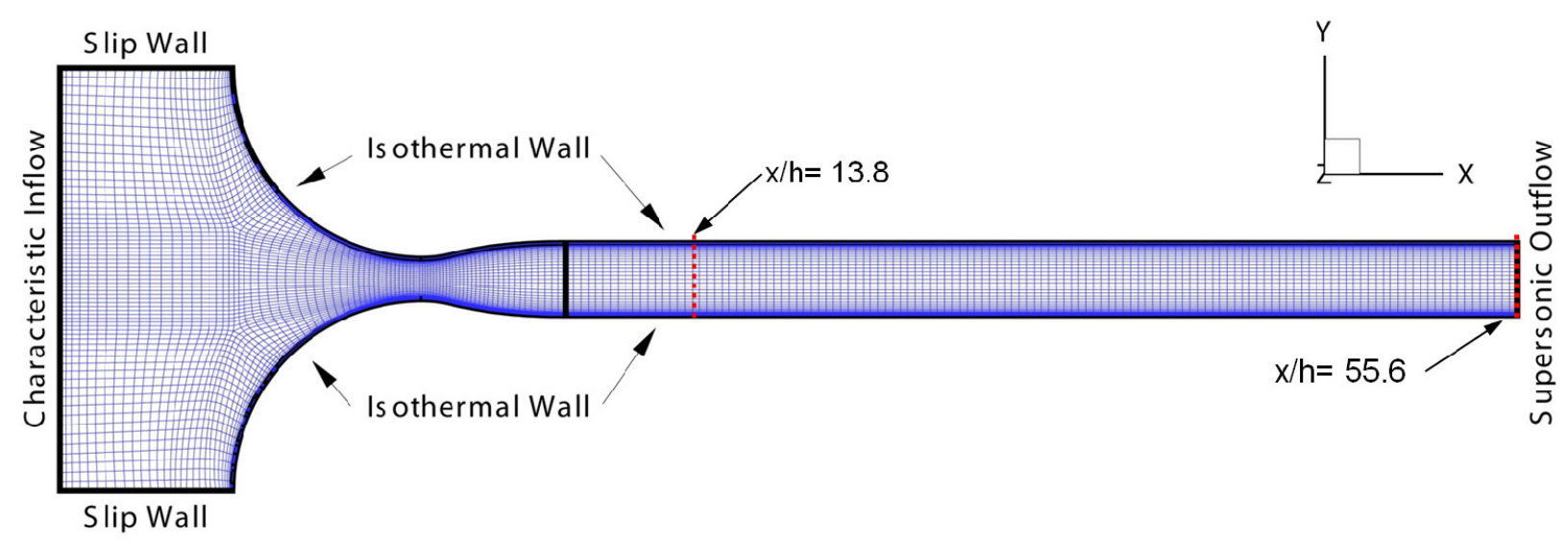

\section{Figure 2. Computational grid}

The CFD simulations of the flowfield were performed using the LAURA solver ${ }^{24}$ developed at the NASA Langley Research Center. The conditions considered involved dry air (mass fractions $\mathrm{N}_{2}$ and $\mathrm{O}_{2}$ of 0.767 and 0.233 respectively) at a stagnation pressure $\left(p_{\text {heater }}\right)$ and temperature $\left(T_{\text {heater }}\right)$ of $296 \mathrm{kPa}$ and $1200 \mathrm{~K}$, respectively. Two simulations were performed, one which assumed thermal equilibrium and one in which thermal non-equilibrium processes were modeled. Simulations for conditions that contained steam were not attempted, due to concerns about whether the modeling assumptions employed for the thermal non-equilibrium processes were valid for steam. Thermal non-equilibrium was modeled using a two-temperature model that assumes that translational and rotational energy modes (with temperatures $T_{\text {trans }}$ and $T_{r}$ ) for each species are in equilibrium, and that the vibrational and electronic energy modes for each species are in equilibrium at temperature $T_{v}{ }^{25}$ The simulations were advanced in pseudo-time using a point-implicit algorithm with a Courant-Friedrichs-Lewy (CFL) number of 5. The inviscid flux components were evaluated using the upwind scheme of Roe ${ }^{26}$ and viscous flux components were evaluated with a centered scheme. The Menter SST model ${ }^{27}$ was chosen to account for flow turbulence. The nozzle flowfield was simulated as a two-dimensional geometry, using the multi-block structured grid shown in Fig. 2. The boundary conditions utilized are also labeled in Fig. 2. The grid topology and resolution was based on the three-dimensional grid employed by researchers at North Carolina State University in their computational studies of this dual-mode scramjet flowpath. The surface temperature was set to $600 \mathrm{~K}$ along the isothermal walls to match that used in these previous computational efforts. ${ }^{28}$

The coordinate system used in this work is also shown in Fig. $2 ; x$ is referenced to the throat of the nozzle; $y$ is referenced to the center of the duct; $z$ is according to a right-handed system and is referenced to the center of the duct.

\section{Results}

\section{A. Existence of Non-equilibrium}

Early analysis of Configuration A CARS spectra acquired in the freestream air of the combustor flow path, conducted while the Configuration A test campaign was still underway, led us to suspect "anomalies": while the rotational band structure of the ground vibrational state of the $\mathrm{N}_{2}$ and $\mathrm{O}_{2}$ Q-branch spectra was consistent with the expected temperature, the first excited vibrational state intensity was too high. It was possible that the observed vibrational non-equilibrium could have been generated by the CARS process itself. This well-known phenomenon is called stimulated Raman pumping (SRP). The green and red laser beams transfer ground-state population to the first excited vibrational state of $\mathrm{N}_{2}$ if the laser energies are sufficiently high via a two-photon process (the left-most up and down arrows in the energy level diagram shown in Fig. 1b). ${ }^{29,30}$ To determine if SRP was affecting the 
measurements, the energy of the green laser $\left(\omega_{p l}\right)$ was varied by a factor of 34 during one run of the experiment. The averages of 200 spectra in each case are shown in Fig. 3 where all the spectra are normalized to equal area under the curve. The horizontal axis is Raman shift $\left(\omega_{p l}-\omega_{S}\right)$ in wavenumbers. If SRP were the cause of this anomaly, the transfer from ground to excited state of $\mathrm{N}_{2}$ should have been changed by a factor of 34 also. Since all the spectra are essentially identical (aside from different levels of instrument noise) we determined that the population distribution is not related to the laser intensity. Thus, SRP is not significant and what we are observing is vibrational non-equilibrium unrelated to the CARS measurement process.

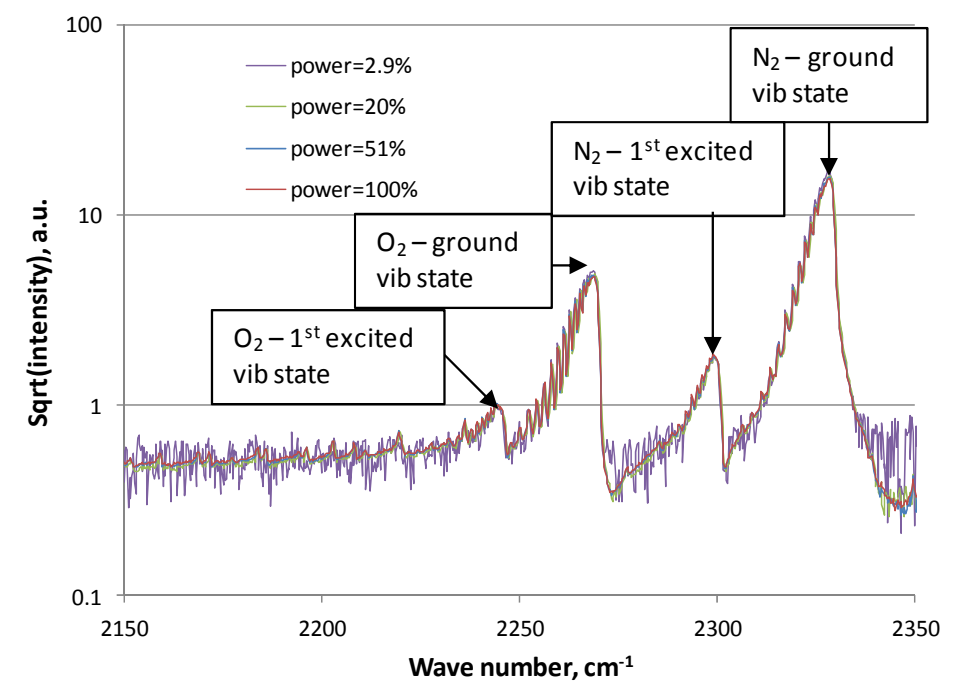

Figure 3. CARS spectra acquired using green laser energies differing by a factor of 34, which was accomplished by rotating the angle of a half wave plate in combination with a polarizer used to attenuate the beam.

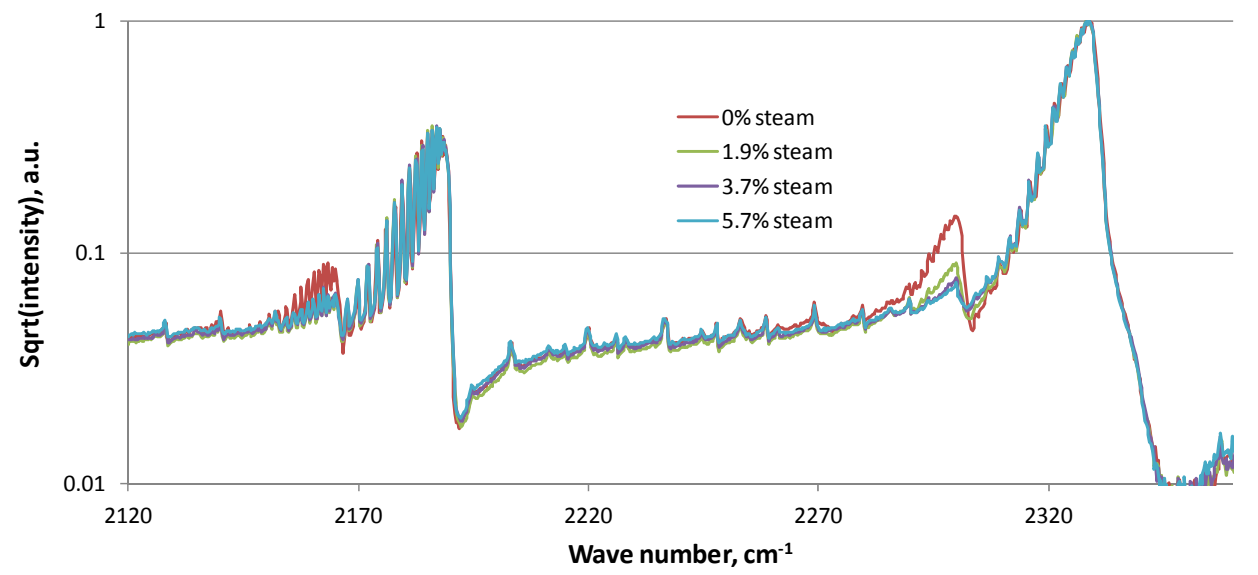

Figure 4. CARS spectra acquired near the center of the duct at $x / h=55.6$ (Configuration C), for constant facility stagnation temperature and pressure but varying mole fractions of steam.

During the Configuration C test campaign (with essentially identical CARS instrumentation to A) we acquired CARS data sets at the entrance plane with various levels of steam introduced in the facility heater. Since steam is known to greatly increase the rate at which $\mathrm{N}_{2}$ relaxes to thermal equilibrium, ${ }^{15}$ this experiment was expected to confirm the hypothesis that non-equilibrium was a result of processes in the flow and provide useful information on relaxation rates. Figure 4 shows experimental CARS spectra (200 shot averages) acquired near the center of the duct for cases with no steam (i.e., "clean air"), and 1.9\%, 3.7\% and 5.7\% steam. The plots are normalized to the peak signal to overlap the rotational band structure of the ground state of $\mathrm{N}_{2}$ for each spectrum. The rotational band 
structure of the ground state of the $\mathrm{N}_{2}$ and the $\mathrm{O}_{2}$ are seen to be identical in all cases, but the height of the first excited vibrational band is reduced by steam, as expected. The spectra for $3.7 \%$ and $5.7 \%$ steam are almost the same, and we assume they are at the equilibrium condition. For $1.9 \%$ the excited band of $\mathrm{N}_{2}$ is a little high, but for $\mathrm{O}_{2}$ it is equilibrium.
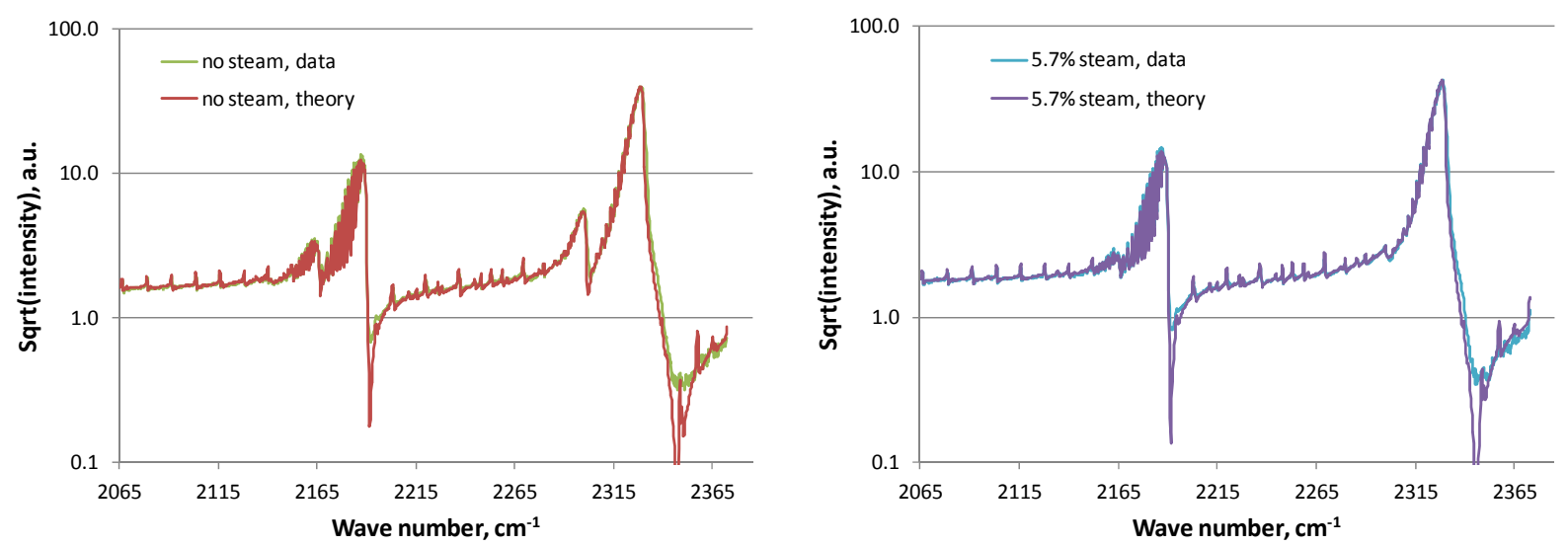

Figure 5. CARS measured spectra and theoretical fits for clean air (left) and 5.7\% steam (right) at the center of the plane for Configuration $\mathrm{C}$. The theory for clean air assumed $T_{v, \mathrm{~N} 2}=1200 \mathrm{~K}, T_{v, 02}=1000 \mathrm{~K}$, and $T_{r}=735$ K. The theory for $5.7 \%$ steam assumed $T_{r}=T_{v}=735 \mathrm{~K}$.

Figure 5 shows fits to theory for these same spectra with no steam and $5.7 \%$ steam using CARSFT. The fits were performed by trial and error variation of rotational and vibrational temperatures, using the known pressure and composition. (This fit with no steam is comparable in appearance and gives the same result within uncertainty to the computed fit described in the next section.) In the case of 5.7\% steam, the best fit occurred with equal rotational and vibrational temperatures $\left(T_{r}=735 \mathrm{~K} \pm 25 \mathrm{~K}\right)$, i.e., with equilibrium, whereas for no steam, different vibrational temperatures for $\mathrm{N}_{2}$ and $\mathrm{O}_{2}$ were found $\left(T_{r}=735 \pm 25 \mathrm{~K}, T_{v, N 2}=1200 \pm 40 \mathrm{~K}, T_{v, O 2}=1000 \pm 40 \mathrm{~K}\right)$. Thus, the vibrational temperature of $\mathrm{N}_{2}$ is frozen at or close to the temperature of the flow exiting the heater (prior to acceleration in the nozzle) while for $\mathrm{O}_{2}$ it is frozen at a somewhat lower temperature. The uncertainties are estimates based again on trial and error and on the sensitivity of the fit to the temperature parameters.

\section{B. Spatial Variation of $T_{r}$ and $T_{v}$ for Clean Air}

The measurement planes for both configuration $\mathrm{A}$ and $\mathrm{C}$ were mapped by acquiring 200 spectra at each of about 170 points on a regular $3 \mathrm{~mm}$ (in $z$ ) $\times 2.4 \mathrm{~mm}$ (in $y$ ) spaced rectangular grid. Some of these grid points lay within the window slots, which were ventilated by cooling air and so registered lower temperatures. In this analysis, the spectra were averaged before fitting. Our library fitting routine is not adapted to fitting vibrational temperature separately from rotational temperature. Our strategy was thus to fit the complete data sets several times with different fixed vibrational temperatures and compare the residuals for each fit. For Configuration C, six libraries were generated, with $T_{v, N 2}=T_{v, O 2}$ of $500,700,900,1100,1300$ and $1500 \mathrm{~K}$. To find the vibrational temperature of $\mathrm{N}_{2}$ at each point, the data set was fitted with each library, blocking from the fits the portion of the spectrum containing the $\mathrm{O}_{2}$ Q-branch (from $2120 \mathrm{~cm}^{-1}$ to $2196 \mathrm{~cm}^{-1}$ ). The fit residual at each point in the grid was fit as a function of temperature to a cubic spline curve and the minimum of this curve was the experimental $T_{v, N 2}$. To find the vibrational temperature of $\mathrm{O}_{2}$ at each point, the procedure was repeated except that the portion of the spectrum containing the $\mathrm{N}_{2}$ Q-branch was blocked $\left(2265 \mathrm{~cm}^{-1}\right.$ to $\left.2352 \mathrm{~cm}^{-1}\right)$. The best rotational temperature at each point was found by generating an additional library using the average fitted $T_{v, N 2}(1200 \mathrm{~K})$ and $T_{v, O 2}(1000 \mathrm{~K})$, and fitting the spectrum for $T_{r}$. This was done three times; first using the complete spectrum, second blocking the $\mathrm{N}_{2} \mathrm{Q}-\mathrm{branch}$, and third blocking the $\mathrm{O}_{2}$ Q-branch. When the $\mathrm{N}_{2}$ was blocked the temperature was $21 \mathrm{~K}$ higher, on average, whereas when the $\mathrm{O}_{2}$ was blocked the temperature was $11 \mathrm{~K}$ lower. The results with no blocking are presented and the spread in temperature is taken as an indication of uncertainty. A similar approach was adopted in analyzing the Configuration A data, differing in some minor details because of differences in the experiment.

The spatial distributions of $T_{v, N 2}, T_{v, O 2}$ and $T_{r}$ are plotted in Fig. 6; the left and right hand column in the figure contain the Configuration A and $\mathrm{C}$ results respectively, while the first, second and third rows contain $T_{v, N 2}, T_{v, O 2}$ and $T_{r}$ respectively. The measurements of $T_{v, O 2}$ for Configuration A are not shown because the precision errors are too 
high to yield meaningful results. All these plots show a deficit in temperature in the top right hand quadrant of the flow. This deficit was previously reported in Ref. 14 for Configuration A, but it is significant that it is still present at the further downstream location in Configuration C. Since this deficit occurs even in the vibrational temperature of $\mathrm{N}_{2}$-which is frozen at close to the facility stagnation condition-the deficit must have existed in the flow before entering the nozzle and be due to non-uniformity in electrical heating of the air. The plot also shows bands of lower temperature at the side walls at $z / h= \pm 3$ caused by the cooling air entering from the window slot. As will be discussed in the next section, the side wall boundary layers are visible in the range $2<|z / h|<2.75$, manifest by an increased rotational temperature (due to viscous heating) and slightly decreased vibrational temperature of $\mathrm{N}_{2}$. The top and bottom wall boundary layer cannot be seen due to lack of data near enough to the walls, which are at $z / h=$ \pm 2 .
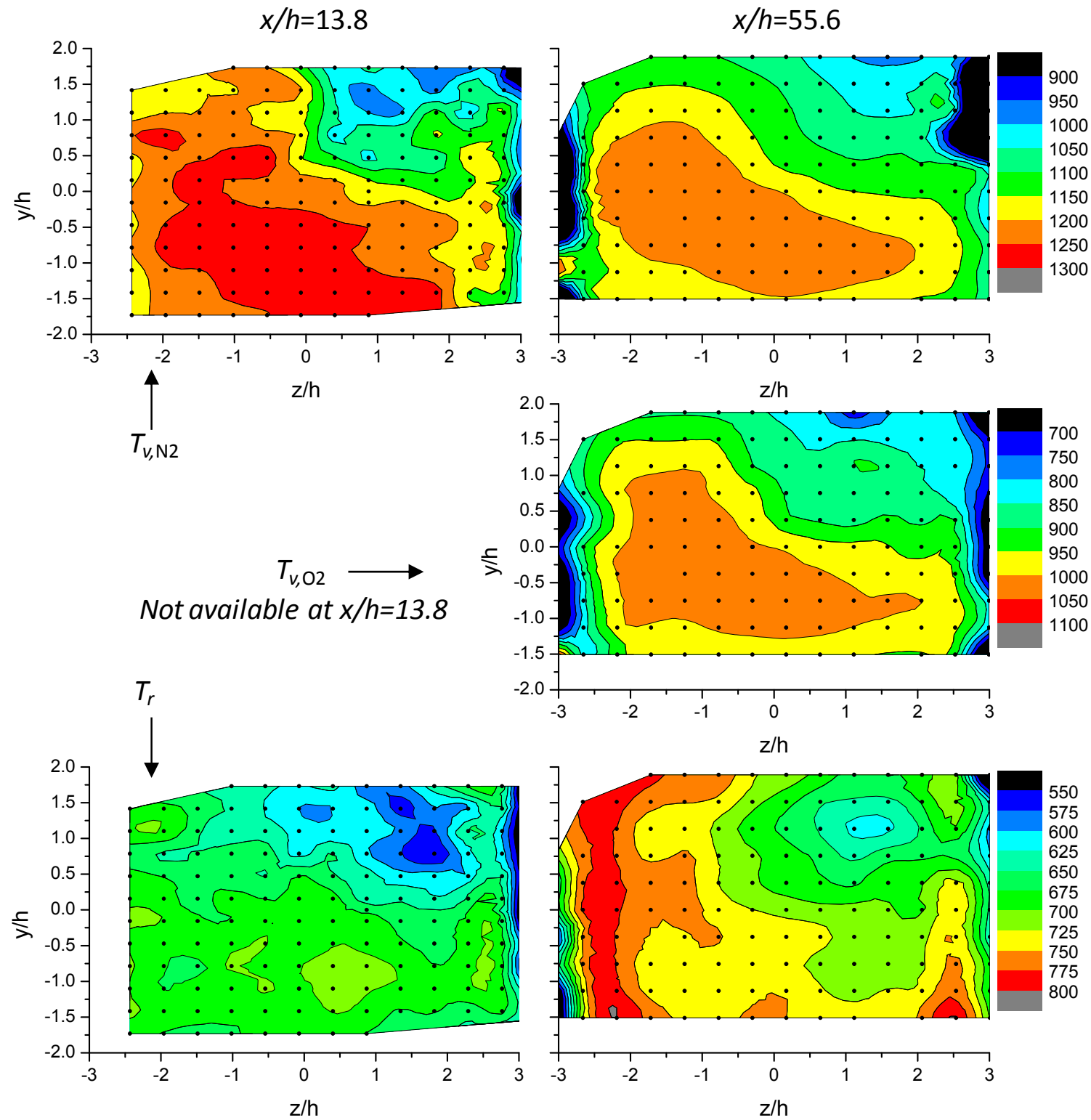

Figure 6. Maps of temperature measured by CARS. The figures on the left are Configuration A, on the right Configuration C. From top to bottom, $T_{v, \mathrm{~N} 2}, T_{v, 02}$, and $T_{r}$. The duct side walls are at $z / h= \pm 3$. Color scales indicated in the legends on the right are consistent left to right but different top to bottom. 


\section{Comparisons of Measured Temperatures with Calculations}

In this section, the results of the computational fluid dynamics calculations using the LAURA code are compared to the CARS measurements. The non-equilibrium simulation computes a translational temperature (equal to the rotational temperature) and a single vibrational temperature, $T_{v}$. To enable the comparisons, a single lumped temperature $T_{v \text {, lump }}$ was calculated from the CARS individually measured $\mathrm{N}_{2}$ and $\mathrm{O}_{2}$ vibrational temperatures by solving Eq. (1).

$$
0.79 E v\left(\theta_{v, \mathrm{~N} 2}, T_{v, \text { lump }}\right)+0.21 E v\left(\theta_{v, 02}, T_{v, \text { lump }}\right)=0.79 E v\left(\theta_{v, \mathrm{~N} 2}, T_{v, \mathrm{~N} 2}\right)+0.21 E v\left(\theta_{v, \mathrm{O} 2}, T_{v, \mathrm{O} 2}\right)
$$

We define $E v=\frac{R \theta_{v}}{\exp \left(\frac{\theta_{v}}{T}\right)}$, where $R$ is the universal gas constant, and $\theta_{v}$ is the characteristic vibrational temperature ( $3390 \mathrm{~K}$ for $\mathrm{N}_{2}$ and $2270 \mathrm{~K}$ for $\mathrm{O}_{2}$ ). ${ }^{1,25}$ For example, given $T_{v, \mathrm{~N} 2}=1200 \mathrm{~K}$, and $T_{v, 02}=1000 \mathrm{~K}$, the solution is $T_{v, \text { lump }}$ $=1148 \mathrm{~K}$.

The temperatures calculated by the LAURA code, assuming non-equilibrium, are plotted against $y / h$ in Fig. 7 , with (a) corresponding to the Configuration A CARS measurement plane and (b) to the Configuration C CARS plane. The lumped vibrational temperature in the freestream is $1140 \mathrm{~K}$ for $\mathrm{A}$ and $1115 \mathrm{~K}$ for $\mathrm{C}$, while the rotational/translational temperature is $662 \mathrm{~K}$ for $\mathrm{A}$ and $691 \mathrm{~K}$ for $\mathrm{C}$. The decrease in vibrational temperature is due to vibrational relaxation while the increase in rotational temperature is mostly due to the growth of boundary layers on the top and bottom wall of the isolator duct, which reduces the Mach number in the freestream, raising temperature and pressure. The calculation is likely to underestimate the actual compression by the boundary layers since it is a 2D calculation while the flow path is rectangular in cross-section (aspect ratio of 1.5) with side wall boundary layers not captured by the calculation. The top and bottom wall boundary layers are visible in the calculation as an increase in rotational temperature approaching the walls and a decrease in vibrational temperature.

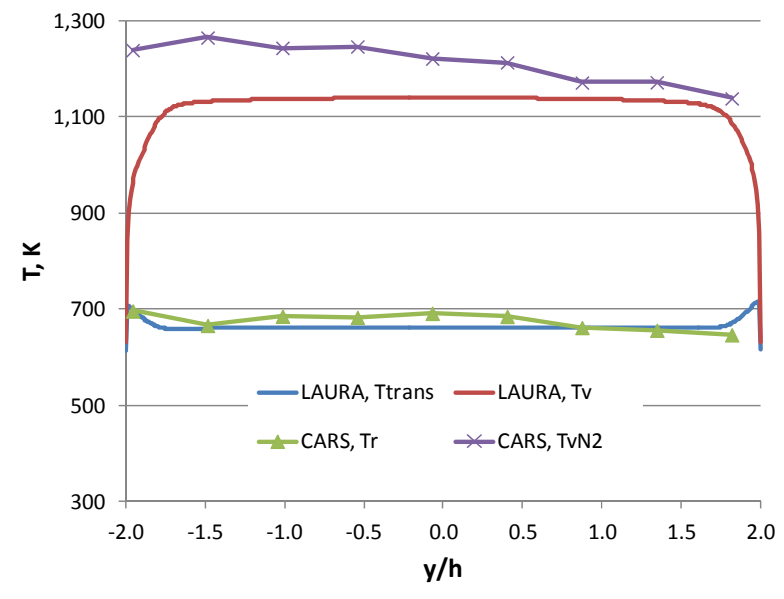

(a)

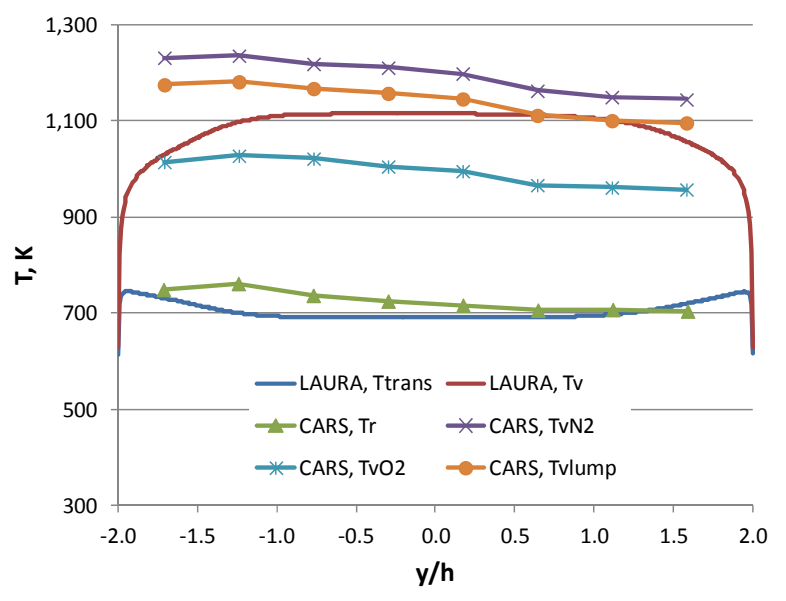

(b)

Figure 7. Profiles of temperature on the center plane $(z=0)$. Comparisons between measured vibrational and rotational temperatures and computational result using the LAURA code. (a) Configuration $A(x / h=13.6)$, (b) Configuration $\mathrm{C}(x / h=55.6)$.

The CARS measured temperatures along the grid line closest to $z=0$ are also shown in Fig. 7, for comparison with the calculation. For Configuration A, despite the previously mentioned spatial non-uniformity in temperature from the heater, the agreement of the measured rotational temperature with calculation is quite good, while the measured $T_{v, \mathrm{~N} 2}$ is greater than the calculated $T_{v, l u m p}$, as expected (since $T_{v, \mathrm{O} 2}$ is less). Notice that $T_{v, \mathrm{~N} 2}>1200 \mathrm{~K}$ where $y<0$, meaning that, in this part of the flow, the temperature entering the nozzle is higher than the nominal facility heater temperature (which was $1200 \mathrm{~K}$ ). For Configuration $\mathrm{C}$, the measured rotational and lumped vibrational temperatures are, on average, a little higher than calculated. This difference is due to the spatial non-uniformity in heater temperature and the growth of the side wall boundary layers, one effect of which is to increase the overall pressure and temperature above that of a $2 \mathrm{D}$ calculation. Like the calculation, the CARS vibrational temperature of $\mathrm{N}_{2}$ is a little less at $\mathrm{C}$ than at A due to vibrational relaxation. The calculation shows an increase in $T_{\text {trans }}$ above the freestream value in the top and bottom wall boundary layers, but a decrease in $T_{v, l u m p}$. As discussed in the previous 
section, this behavior is not seen in the CARS data (shown in Fig. 6) near the top and bottom walls due to lack of measurements there, but something similar is seen near the side walls.

\section{Effect of Steam on Wall Pressure and Flow Temperature}

Figure 8 shows measured and calculated wall static pressures, normalized by the facility heater pressure and plotted as a function of distance down the duct $(x / h)$. The plot includes measurements with clean air and steam, as well as calculations assuming non-equilibrium and equilibrium. As the distance increases, the calculated pressure drops within the nozzle to a minimum at the nozzle exit, then rises gradually in the isolator due to growth of top and bottom wall boundary layers. For clean air, the measured pressure at the first point (after the nozzle exit) is in agreement with the non-equilibrium calculation, as is the general trend of increasing pressure in the isolator. However, there are oscillations in the measurements not observed in the calculations, presumably due to reflecting shock waves. The average slope of the measurement is a little higher than the slope of the calculation due to side wall boundary layers not reproduced by the $2 \mathrm{D}$ calculation. The effect of steam is to raise the pressure at the nozzle exit by from $4.5 \%$ (for $1.9 \%$ steam) to $6.4 \%$ (for $5.7 \%$ steam) and this difference is maintained throughout the isolator. Part of this change is due to a change in thermodynamic properties, but this effect is small as evidenced by the small change between the measurements as the percentage of steam is varied. On the other hand, the change due to steam is reproduced by switching the calculation from non-equilibrium to thermal equilibrium, consistent with the CARS observation that addition of steam brings the system towards equilibrium.

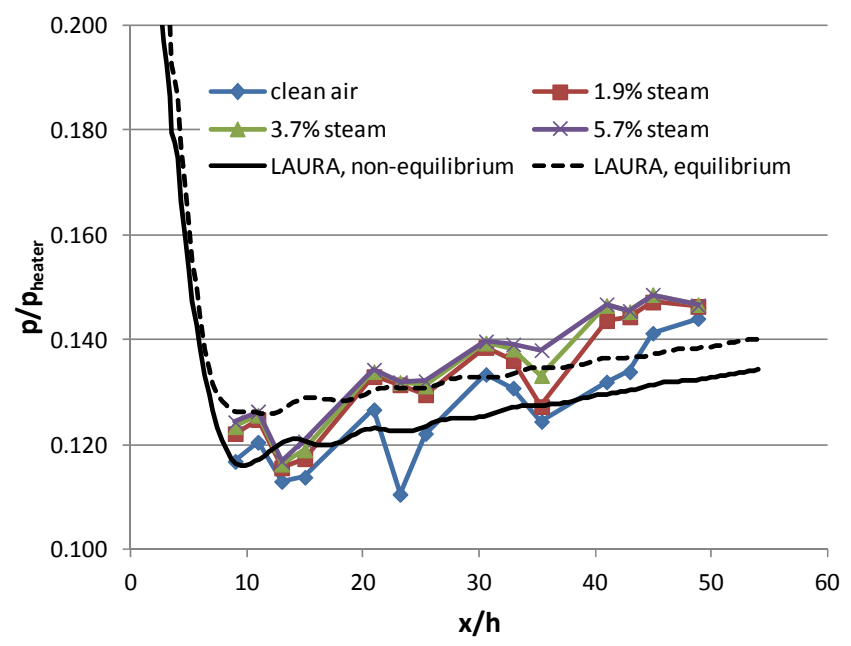

Figure 8. Pressure on the interior flow surface of the flow path $(z=0)$ : measured pressure for clean air and several steam levels, and computational results for non-equilibrium and equilibrium.

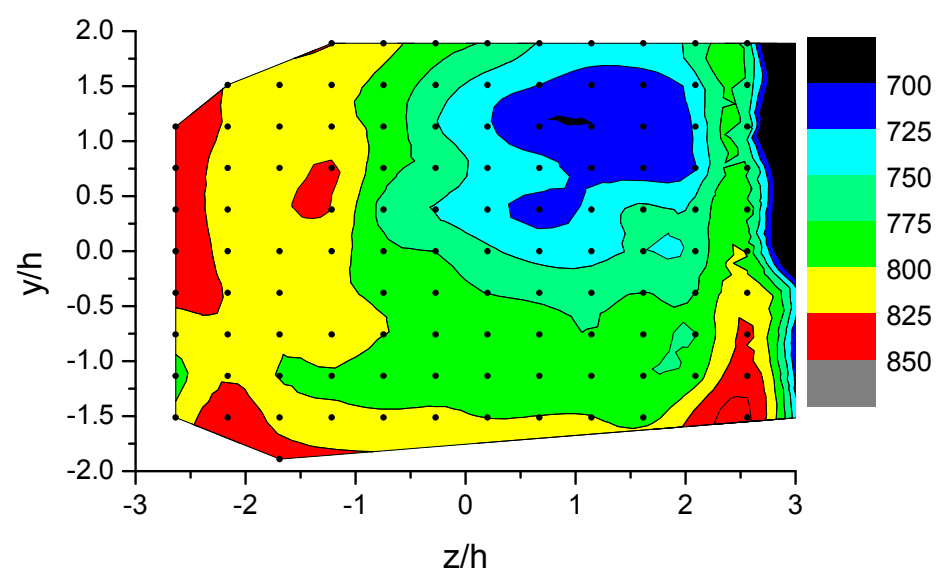

Figure 9. Map of CARS rotational temperature for Configuration $C$ with $1.9 \%$ steam 
Figure 9 shows a map of the CARS temperature for Configuration $\mathrm{C}, 1.9 \%$ steam, spectra fitted assuming $T_{v, \mathrm{~N} 2}=$ $950 \mathrm{~K}$ and thermal equilibrium of $\mathrm{O}_{2}$. The temperature distribution in the map is generally similar to the distribution of rotational temperature for Configuration $\mathrm{C}$, clean air shown in Fig. 6, although temperatures are on average about $44 \mathrm{~K}$ (about 6\%) higher. The CARS results at $z=0$ are also shown in Fig. 10 as a function of $y / h$ and compared to the measurements for clean air and the calculations. The measured increase in temperature from clean air to $1.9 \%$ steam is accurately reproduced by the calculated increase from non-equilibrium to equilibrium, although the overall level of the calculation is low due to the neglect of the side wall boundary layers.

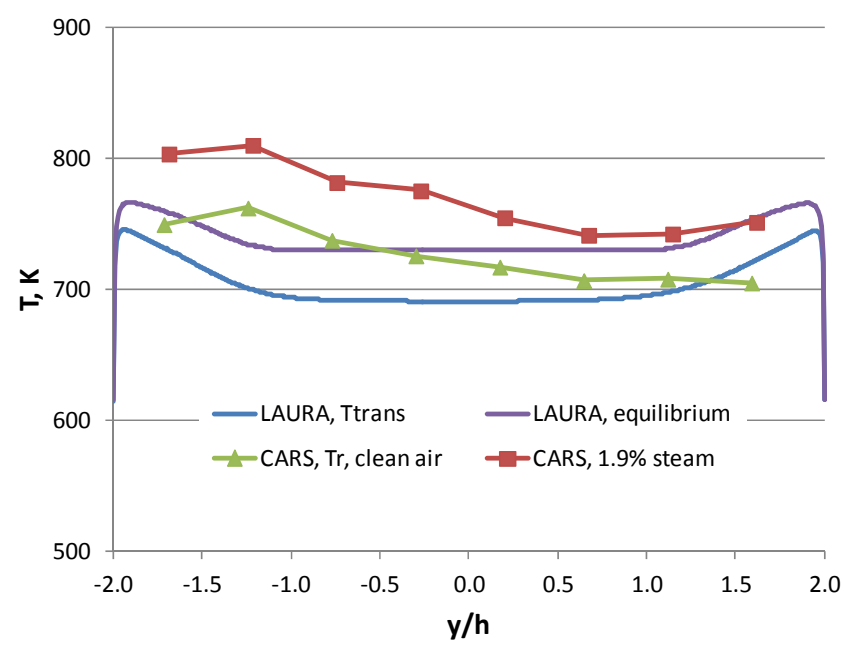

Figure 10. Profiles of temperature on the center plane for Configuration C. CARS measurements with clean air and $1.9 \%$ steam. Computational results for non-equilibrium and equilibrium.

\section{Discussion and Conclusions}

In this work, we have measured the vibrational and rotational temperatures in two planes in a constant area duct mounted to the exit of a Mach 2 nozzle at the University of Virginia Supersonic Combustion Facility. With clean air flowing in the facility at a nominal stagnation temperature of $1200 \mathrm{~K}$, we have measured non-equilibrium vibrational temperatures of $\mathrm{N}_{2}$ and $\mathrm{O}_{2}$. Indeed, the $\mathrm{N}_{2}$ vibrational temperature of about $1200 \mathrm{~K}$ was close to the facility heater stagnation temperature, while the vibrational temperature of $\mathrm{O}_{2}$ was little lower $(1000 \mathrm{~K})$. This result was at first a surprise since, although vibrational freezing is commonly encountered in hypersonic nozzles, it is not typically expected in supersonic ones. The existence of vibrational freezing is a function of the particular operating conditions. Supersonic wind tunnels are not usually operated with heated air while hypersonic wind tunnels typically are, so vibrational modes of energy are simply not populated for supersonic nozzle flows. On the other hand, since the time required to establish thermal equilibrium grows steeply with decreasing temperature (and pressure), ${ }^{1}$ for a higher heater temperature, the air might not drop below the threshold in temperature at which freezing occurs. The relatively small scale of the facility is also a factor. Computational fluid dynamics calculations using established models for vibrational relaxation were able to predict the rotational and "lumped" vibrational temperatures. Measurements were also made in the facility with several molar percentages of steam $(1.9 \%, 3.7 \%$ and 5.7\%). Steam was found to bring the flow to thermal equilibrium, consistent with expectations based on the effect of steam on relaxation rates known from the literature. Steam significantly increases both temperature and pressure at the nozzle exit due to the change from vibrationally frozen to thermal equilibrium flow. This work also confirms previous observations of non-uniformities of temperature entering the facility nozzle, and shows that this non-uniformity persists at least to the end of the facility isolator. Both vibrational non-equilibrium and temperature non-uniformity are expected to affect the distribution of pressure and combustion in the scramjet flow path. This greater understanding of the flow in the nozzle and isolator will substantially assist our interpretation of measurements acquired in the dual-mode scramjet combustor experiments being conducted in the facility.

\section{Acknowledgments}

We would like to thank Roger Reynolds (University of Virginia) for his support and operation of the wind tunnel. This research was sponsored by the National Center for Hypersonic Combined Cycle Propulsion grant FA 
9550-09-1-0611. The technical monitors on the grant are Chiping Li (AFOSR) and Rick Gaffney (NASA). Support for Paul Danehy was provided by NASA's Fundamental Aeronautics Program, Hypersonics Project, Propulsion Discipline.

\section{References}

${ }^{1}$ Vincenti W.G., Kruger, C.H., Introduction to physical gas dynamics, John Wiley, New York, 1965.

${ }^{2}$ Sharma, S.P., Ruffin, S.M., Gillespie, W.D., Meyer, S.A., "Vibrational relaxation measurements in an expanding flow using spontaneous Raman scattering", Journal of Thermophysics and Heat Transfer, Vol. 7:4, 1993, pp. 697703.

${ }^{3}$ Palma, P.C., Danehy, P.M., Houwing, A.F.P., "Fluorescence Imaging of Rotational and Vibrational Temperature in a Shock Tunnel Nozzle Flow," AIAA Journal, Vol. 41, No. 9, Sept. 2003, pp. 1722-1732.

${ }^{4}$ Fraval, E., Danehy, P.M., Houwing, A.F.P., "Single-Shot Broadband Coherent Anti-Stokes Raman Scattering Measurements in a Free Piston Shock Tunnel Nozzle Expansion," in Proceedings of $23^{\text {rd }}$ International Symposium on Shock Waves, Fort Worth, Texas, Published by The University of Texas at Arlington, Arlington, Texas, USA. (CD ROM), pap. num. 1717, July 22-27, 2001, pp. 396-402.

${ }^{5}$ Nompelis, I., Candler, G.V., Holden, M.S., "Effect of Vibrational Non-equilibrium on Hypersonic Double-Cone Experiments," AIAA Journal, Vol. 41, No. 11, 2003, pp. 2162-2169.

${ }^{6}$ Millikan, R.C., White, D.R., "Systematics of vibrational relaxation", Journal of Chemical Physics, Vol. 139, 1963, pp. 3209-3213.

${ }^{7}$ Sebacher, D. I.; Guy, R. W., "Vibrational relaxation in expanding $\mathrm{N}_{2}$ and air," NASA-TM-X-71988, 1974.

${ }^{8}$ J. C. Hoppe "Rotational and vibrational temperature measurements in the 12-inch hypersonic ceramic-heated tunnel", NASA-TN-D-4892, Nov 1, 1968.

9 Lucht R. P., "Three laser Coherent Anti-Stokes Raman Scattering Measurements of Two Species," Opt. Lett., Vol. 12, 1987, pp. 78-80.

${ }^{10}$ Cutler A. D., Danehy P. M., Springer R. R., O’Byrne S., Capriotti D. P., and Deloach R., "Coherent Anti-Stokes Raman Spectroscopy Thermometry in a Supersonic Combustor," AIAA J., Vol. 41, No. 12, Dec 2003, pp. 24512459.

${ }^{11}$ Anderson T. J., and Eckbreth A. C., "Simultaneous Coherent Anti-Stokes Raman Spectroscopy Measurements in Hydrogen Fueled Supersonic Combustion,” J. Propul. Power, Vol. 8, No. 1, Jan 1992, pp. 7-15.

${ }^{12}$ Yang S. R., Zhau J. R., Sung G. J., and Yu G., "Multiplex CARS Measurements in Supersonic Hydrogen/Air Combustion," Appl. Phys. B, Vol. 68, No. 2, 1999, pp. 257-265.

${ }^{13}$ O’Byrne S., Danehy P. M., Tedder S. A., and Cutler A. D., "Dual pump Coherent Anti-Stokes Raman Scattering Measurements in a Supersonic Combustor", AIAA J., Vol. 45, No. 4, April 1997, pp. 922-933.

${ }^{14}$ Cutler, A.D., Magnotti, G., Cantu, L., Gallo, E., Danehy, P.M., Rockwell, R.D., Goyne, C.P., McDaniel, J.C., "Dual-Pump CARS Measurements in the University of Virginia's Dual-Mode Scramjet: Configuration "A"," AIAA2012-0114, 50 $0^{\text {th }}$ AIAA Aerospace Sciences Meeting and Exhibit, Nashville, TN, 2012.

${ }^{15}$ Center, R.E., Newton, J.F., "Vibrational Relaxation of $\mathrm{N}_{2}$ by $\mathrm{H}_{2} \mathrm{O}$," J. Chem Phys., Vol. 68, No. 8, April 1978.

${ }^{16}$ Rockwell, R. D., Jr., Goyne, C. P., Haw, W., Krauss, R. H., McDaniel, J. C., and Trefny, C. J., "Experimental Study of the Effects of Test Media Vitiation on the Performance and Operation of a Dual-Mode Scramjet," Journal of Propulsion and Power, Vol. 27, No. 5, 2011, pp. 1135-1142.

${ }^{17}$ Goyne, C. P., McDaniel, J. C., Krauss, R. H., and Whitehurst, W. B., "Test Gas Vitiation Effects in a Dual-mode Scramjet Combustor," Journal of Propulsion and Power, Vol. 23, No. 3, 2007, pp. 559-565.

${ }^{18}$ Rockwell, R.D., Goyne, C.P., Haw, W., McDaniel, J.C., Goldenstein, C.S., Schultz, I.A., Jeffries, J.B., and Hanson, R.K., "Measurement of water vapor levels for investigating vitiation effects on scramjet performance," Journal of Propulsion and Power, Vol. 27, No. 6, 2011, pp. 1315-1317.

${ }^{19}$ Magnotti, G., Cutler, A.D., Danehy, P.M., "Development of a Dual-Pump CARS System for Measurements in a Supersonic Combusting Free Jet," AIAA-2012-1193, 50 ${ }^{\text {th }}$ AIAA Aerospace Sciences Meeting, Nashville, TN, January, 2012.

${ }^{20}$ Magnotti, G., Cutler, A. D., and Danehy, P. M. "Beam Shaping for CARS Measurements in Turbulent Environments," AIAA-2010-1400 48 ${ }^{\text {th }}$ AIAA Aerospace Sciences Meeting, Orlando, FL, 2010

${ }^{21}$ Palmer R. E., "The CARSFT Computer Code for Calculating Coherent Anti-Stokes Raman Spectra: User and Programmer Information”, Sandia Report: Sand89:8206,1989. 
${ }^{22}$ Cutler A.D., Magnotti G., "CARS Spectral Fitting with Multiple Resonant Species Using Sparse Libraries “,AIAA-2010-1400, 48 ${ }^{\text {th }}$ AIAA Aerospace Sciences Meeting, Orlando, FL, January, 2010.

${ }^{23}$ Varghese, P.L., Hanson, R.K., Appl. Opt., Vol. 23, No. 14, 1984, pp. 2376-2385.

${ }^{24}$ Mazaheri, A., Gnoffo, P.A., Johnston, C.O., Kleb, B., LAURA Users Manual: 5.4-54166, NASA TM 217092, 2011.

${ }^{25}$ Gnoffo, P.A., Gupta, R.N., Shinn, J.L., "Conservation Equations and Physical Models for Hypersonic Air Flows in Thermal and Chemical Non-equilibrium," NASA TP 2867, 1989.

26 Roe, P.L., "Approximate Riemann Solvers, Parameter Vectors and Difference Schemes," Journal of Computational Physics, Vol. 43, 1981, pp. 357-372.

${ }^{27}$ Menter, F.R., Kuntz, M., Langtry, R., "Ten Years of Industrial Experience with the SST Turbulence Model," Turbulence, Heat and Mass Transfer 4, ed: Kanjalic, Y. Nagano, and M. Tummers, Begell House, Inc. 2003, pp. 625-632.

${ }^{28}$ Fulton, J.A., Edwards, J.R., Hassan, H.A., Rockwell, R.D., Goyne, C.P., McDaniel, J.C., Smith, C., Cutler, A.D., Johansen, C., Danehy, P.M., Kouchi, T., "Large-eddy / Reynolds-averaged Navier-Stokes Simulation of a DualMode Scramjet Combustor," AIAA-2012-0115, 50 ${ }^{\text {th }}$ AIAA Aerospace Sciences Meeting, Nashville, TN, January, 2012.

${ }^{29}$ Gierulski A., Noda M., Yamamoto T., Marowsky G., Slenczka A., "Pump-induced population changes in broadband coherent anti-Stokes Raman scattering”, Opt. Lett., Vol. 12, Iss. 8, 1987, pp.608-610.

${ }^{30}$ Magnotti, G., Cutler, A.D., Tedder, S.A., Herring, G.C., Danehy, P.M., "Saturation and Stark Broadening Effects in Dual-Pump CARS of $\mathrm{N}_{2}, \mathrm{O}_{2}$ and $\mathrm{H}_{2}$," J. of Raman Spectroscopy, Vol. 43, Iss. 5, pp. 611-620, 2012 